\title{
Absolute quantification in multi-pinhole micro-SPECT for different isotopes
}

\author{
B. Vandeghinste, Student Member, IEEE, C. Vanhove, J. De Beenhouwer, R. Van Holen, Member, IEEE, \\ S. Vandenberghe, and S. Staelens
}

\begin{abstract}
In preclinical Single Photon Emission Tomography (SPECT), absolute quantification is interesting, expressed in percentage of injected radioactive dose per gram of tissue. This allows for accurate evaluation of disease progression and precise follow-up studies without the need for sacrificing animals. Accurate modeling of image degrading effects is currently under development for isotopes different from ${ }^{99 m}$ Tc.

The aim of this work is to develop absolute micro-SPECT quantification for three different isotopes: ${ }^{99 m} \mathbf{T c},{ }^{111} \mathbf{I n}$ and ${ }^{125} \mathbf{I}$. This selection of isotopes covers a wide range of energies, is pre-clinically relevant and allows us to optimally validate the algorithms used for image reconstruction. Furthermore, we will mix these isotopes with additional iodine-based CT contrast agent, to mimic contrast-enhanced SPECT/CT protocols.

For each isotope, both a calibration phantom and three 1ml vials were scanned on the CZT-based FLEX Triumph-I scanner (GM-I). The calibration phantom allows the conversion of reconstructed voxel counts to $\mathrm{MBq} / \mathrm{ml}$. The 3-vial phantom consists of 3 different concentrations of radioactivity. Two vials contain iodine-based CT contrast agent to significantly increase the attenuation. All datasets were reconstructed using a GPUbased reconstruction algorithm, which includes resolution recovery, pinhole penetration, geometrical sensitivity correction, scatter correction and attenuation correction.

We show good quantification for ${ }^{99 m} \mathbf{T c}$ and ${ }^{111} \mathbf{I n}$. The absolute quantification of ${ }^{125} I$ is suboptimal, due to insufficient scatter correction. No influence can be seen when iodine-based CT contrast agent is used together with ${ }^{125} \mathrm{I}$.
\end{abstract}

\section{INTRODUCTION}

$\mathbf{I}$ $\mathrm{N}$ vivo pre-clinical micro Single Photon Emission Computed Tomography (SPECT) allows researchers to visualize pharmacological and physiological processes on a molecular level. This is an aid in research on oncological, cardiovascular and neurological diseases and allows the validation of therapies in a pre-clinical phase.

Manuscript received November 10, 2011. This work was supported in part by a PhD grant to Bert Vandeghinste of the Institute for the Promotion of Innovation through Science and Technology in Flanders (IWT-Vlaanderen), by a postdoctoral fellowship of the Research Foundation Flanders (FWO) to Roel Van Holen, and by the CIMI project, an IBBT-project in cooperation with: Barco nv, DCILABS, IBA Dosimetry, GE and DSC Labs. IBBT is an independent multidisciplinary research institute founded by the Flemish Government, to stimulate ICT innovation. Chris Vanhove is supported by the GROUP-ID consortium from Ghent University.

Bert Vandeghinste, Chris Vanhove, Jan De Beenhouwer, Roel Van Holen, Stefaan Vandenberghe and Steven Staelens are with the Department of Electronics and Information Systems, MEDISIP, Ghent University-IBBTIBiTech, De Pintelaan 185 block B, B-9000 Ghent, Belgium (e-mail: bert.vandeghinste@ugent.be).

J. De Beenhouwer is also with The Vision Lab, University of Antwerp, 2610 Wilrijk, Belgium.

S. Staelens is also with the Molecular Imaging Centre Antwerp, University of Antwerp, 2650 Edegem, Belgium.
We are mostly interested in absolute SPECT quantification in pre-clinical research, expressed in percentage of injected radioactive dose per gram of tissue. A number of benefits can then be gained such as modeling the kinetics of the radioligand specific binding, the receptor density and relevant biochemical parameters [1]. This leads to faster drug development, better treatment monitoring, improved follow-up and reduced variability in experimental design, as the animals can serve as their own control when sacrificing is not needed anymore.

The different errors leading to inaccurate quantification can be attributed to photon attenuation, photon scattering, partial volume effect (PVE) and system imperfections. These systematic imperfections can be compensated for by modeling this effect during image reconstruction. The most common approach is to measure the system response at a grid of discrete locations in the field of view (FOV) of the camera. This system response accounts for geometric response together with more complex effects such as detector variability and collimator imperfections. A different approach is to directly incorporate the physical processes leading to these effects (e.g. detector response, limited pinhole diameter, sensitivity) into ray-driven reconstruction. The more accurate forward projection operator will then provide projections closer to the measured data. Absolute quantification errors from $8 \%$ to $11 \%$ have been reported for ${ }^{111} \mathrm{In}$ and from $+4 \%$ to $+10 \%$ for ${ }^{99 m} \mathrm{Tc}$ with raydriven methods [2], [3]. An error of $-6.3 \%$ to $+4.3 \%$ for ${ }^{99 m} \mathrm{Tc}$ has only been obtained using system matrix measurements [4]. Accurate modeling of image degrading effects is for different isotopes still needs improvement.

The aim of this work is to develop absolute quantitative micro-SPECT methods for three different isotopes: ${ }^{99 m} \mathrm{Tc}$ (traditional micro-SPECT imaging), ${ }^{111}$ In (longer half-life for radiolabeling antibodies) and ${ }^{125}$ I (stem cell trafficking). This selection of isotopes allows us to optimally validate the software and is pre-clinically relevant.

To our knowledge, absolute quantification errors below these reported by Finucane et al. [2] and Vanhove et al. [3] have not been reported for preclinical imaging with raybased methods. Furthermore, the error in case of simultaneous contrast agent and radioactive tracer has not been reported for ${ }^{125} \mathrm{I}$.

\section{Materials AND Methods}

\section{A. SPECT/CT System}

The FLEX Triumph-I system (Gamma-Medica Ideas, Northridge, CA, USA) was used to acquire SPECT projection 


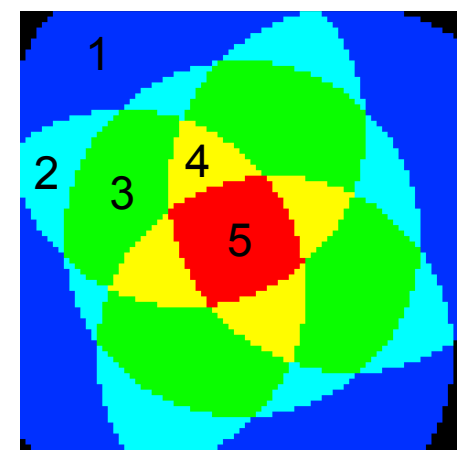

Fig. 1: Projections overlap for one detector, annotated with the amount of overlapping pinholes in each region.

images. This tri-modal preclinical system consists of four 80by-80 pixels CZT detector heads with $1.6 \mathrm{~mm}$ pixel size, each equiped with a multi-pinhole collimator. Each multi-pinhole collimator and consists of 5 tilted pinholes with $0.5 \mathrm{~mm}$ nominal diameters. The collimator detector distance is $75 \mathrm{~mm}$, the radius of rotation can be varied and was set to $45 \mathrm{~mm}$ for these studies. This leads to a large overlap between individual pinhole projections. This is depicted on the color coded Fig. 1. The energy resolution of the pixelated CZT detector is $9 \%$ for $140.5 \mathrm{keV}\left({ }^{99 m} \mathrm{Tc}\right)$ and $32 \%$ for $27.5 \mathrm{keV}\left({ }^{125} \mathrm{I}\right)$ [7]. We did not measure the energy resolution for ${ }^{111} \mathrm{In}$.

The methods developed by Bequé et al. [8], [9] were used to calibrate the SPECT geometry. The measured projections from three ${ }^{99 m}$ Tc point sources embedded in an acrylic cylindrical phantom were automatically segmented and analyzed to find the 15 centroids per projection angle per detector head. All centroids were then used in an optimization scheme, resulting in focal length and the location for each pinhole, distance between detector and rotation axis, offset of detector center, detector tilt and skew and the angle offset between the 4 detector heads. Relevant geometrical parameters are shown in Table I.

All SPECT acquisitions were acquired using 64 projection views over 360 degrees, with a 1-minute exposure time per view.

Micro-CT acquisitions followed the SPECT acquisitions and were measured with the CT of the FLEX Triumph system, located on the same gantry as the SPECT, leading to optimal co-registration. The CT images were acquired using 1024 projections, detected by a 2368 x 2240 detector with $50 \mu \mathrm{m}$ pixel size. The X-ray tube has a nominal focal spot of $50 \mu \mathrm{m}$ and produced a tube current of $285 \mu \mathrm{A}$ at $75 \mathrm{kVp}$. The total acquisition time was 8.5 minutes.

The micro-CT data was reconstructed analytically to a $512 \times$ $512 \times 512$ voxel space with an isometric voxel size of $115 \times$ $115 \times 115 \mu \mathrm{m}^{3}$. The data was then smoothed with a $2^{3}$-pixel median filter and linearly scaled to narrow-beam attenuation values (no implicit scatter correction) at photopeak energy for ${ }^{99 m} \mathrm{Tc}$ and ${ }^{111} \mathrm{In}$, and to broad-beam attenuation values (to correct for some portion of the scatter) for ${ }^{125} \mathrm{I}$.
TABLE II: Activity concentrations of the three 1-ml vials in $\mathrm{MBq} / \mathrm{ml}$.

\begin{tabular}{|l|c|c|c|}
\hline & $1: 4$ & $1: 2$ & $1: 1$ \\
\hline${ }^{99 m} \mathrm{Tc}$ & 67.35 & 124.74 & 230.42 \\
${ }^{125} \mathrm{I}$ & 17.01 & 30.19 & 66.37 \\
${ }^{111} \mathrm{In}$ & 5.08 & 8.29 & 14.47 \\
\hline
\end{tabular}

\section{B. Phantom acquisitions}

For each isotope, two phantoms were made and scanned. The first phantom (Fig. 2a) was a calibration source, made by uniformly filling a syringe with known activity: $66.29 \mathrm{MBq}$ for ${ }^{99 m} \mathrm{Tc}, 17.69 \mathrm{MBq}$ for ${ }^{125} \mathrm{I}$ and $40.00 \mathrm{MBq}$ for ${ }^{111} \mathrm{In}$. This allows us to relate the number of counts in reconstructed images to the absolute activity.

The second phantom (Fig. 2b) consists of three 1-ml vials, which have been filled with 3 different activity concentrations. Table II shows the exact activity concentrations for each isotope. The total diameter of the phantom is about $30 \mathrm{~mm}$, comparable with the diameter of lean mice. $0.25 \mathrm{ml}$ iodinebased contrast agent (Visipaque $320 \mathrm{mgI} / \mathrm{ml}$, GE Healthcare) was added as part of the dilution mixture to the $1: 2$ and 1:4 vials, to investigate the influence of iodine-based contrast agents on SPECT imaging.

\section{Quantitative SPECT reconstruction}

1) Listmode processing: The SPECT listmode output was first calibrated to the correct photopeak energy, corrected for pixel uniformity and then converted to sinogram data. Pixel uniformity was measured for each isotope using a low-count point source located $170 \mathrm{~mm}$ from uncollimated detectors. All data was corrected for decay and normalized to total acquisition time.

2) Scatter correction: The photon scatter was derived from the listmode data using Triple Energy Window correction (TEW) for ${ }^{99 m} \mathrm{Tc}$, with a $14 \mathrm{keV}$ photopeak window and $3 \%$ scatter energy windows (SEWs) [10]. Five Energy Window correction (FEW) was used for ${ }^{111}$ In, using a $20 \%$ photopeak window for both photopeaks and 8\% SEWs [11]. No scatter correction was implemented for ${ }^{125} \mathrm{I}$, because the energy resolution of $32 \%$ at $27.5 \mathrm{keV}$ is insufficient to measure the scatter fraction in the photopeak.

The absolute number of scattered photons was integrated and stored in sinogram space, then filtered using a $3 \times 3$ pixel median filter followed by a fourth-order Butterworth filter with a cutoff frequency of 0.127 cycles/cm to suppress noise amplification in the corrected images [16]. This sinogram was then added to the forward projection estimate, before comparing with the measured sinogram.

3) Attenuation correction: Attenuation correction was computed directly during the forward projecting step by applying Beer's Law while forward projecting along each ray. Speed-up is achieved by using 2D texture interpolation on the projections and $3 \mathrm{D}$ texture interpolation on the image estimate and the attenuation map. In this way, no down sampling is needed to obtain an equally sized attenuation map and reconstructed image. This reconstruction package was implemented in CUDA, 
TABLE I: Geometrical parameters determined by calibration. The focal length is reported as mean \pm SD over 5 pinholes.

\begin{tabular}{|l|c|c|c|c|}
\hline & Head 1 & Head 2 & Head 3 & Head 4 \\
\hline Focal Length (mm) & $75.597 \pm 0.105$ & $76.057 \pm 0.345$ & $77.211 \pm 0.040$ & $76.953 \pm 0.141$ \\
Detector - Rotation axis (mm) & 112.205 & 112.964 & 107.918 & 108.083 \\
Tilt (degrees) & 0.909 & 3.370 & 2.073 & 3.385 \\
Skew (degrees) & 0.237 & 0.248 & -0.273 & 0.029 \\
offset (u, v) (mm) & $(-6.814,0.505)$ & $(3.370,0.248)$ & $(-4.792,3.869)$ & $(-0.479,6.222)$ \\
Starting angle (degrees) & 0 & 183.282 & 91.564 & 273.108 \\
\hline
\end{tabular}
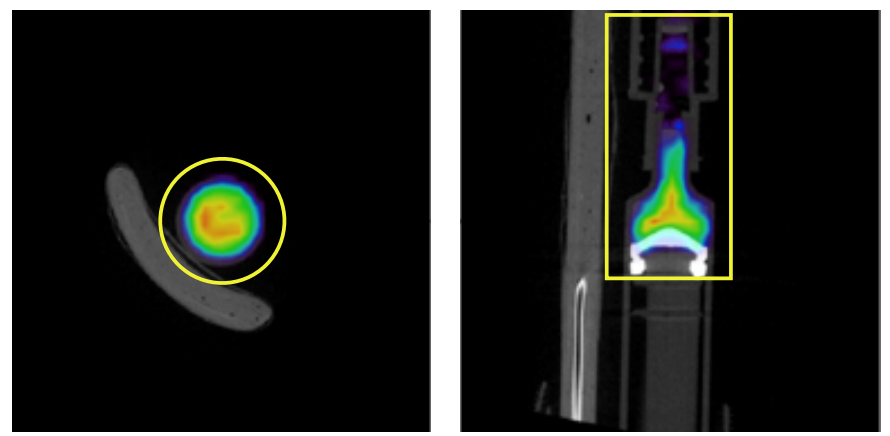

(a)
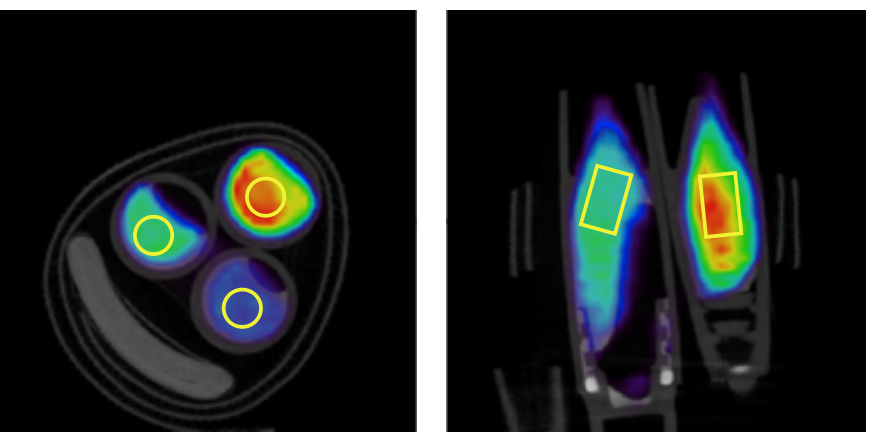

(b)

Fig. 2: (a) Reconstruction of the calibration syringe for ${ }^{99 m} \mathrm{Tc}$. (b) Reconstructed image of the 3-vial phantom superimposed on the attenuation map.

and runs on one Tesla M2070 GPU (NVIDIA, Santa Clara, CA, USA).

4) Pinhole penetration: Pinhole penetration is taken into account for each specific photopeak by enlarging the nominal pinhole diameter to the effective diameter $d_{s}$ for sensitivity calculations and $d_{r}$ for resolution recovery:

$$
\begin{aligned}
d_{s} & =\sqrt{d\left(d+\frac{2}{\mu} \tan \frac{\alpha}{2}\right)}+\frac{2}{\mu^{2}} \tan ^{2} \frac{\alpha}{2} \\
d_{r} & =\sqrt{d\left(d+\frac{2}{\mu} \tan \frac{\alpha}{2}\right)},
\end{aligned}
$$

with $d$ the nominal pinhole diameter, $\mu$ the linear attenuation coefficient of its material (here Tungsten) and $\alpha$ the full acceptance angle of the pinhole [13], [14].

5) Sensitivity: Geometrical sensitivity was corrected analytically by calculating the sensitivity $g$ of a pinhole as

$$
g=\frac{d_{s}^{2} \sin ^{3} \theta}{16 h^{2}}
$$

where $\theta$ is the incidence angle measured from the plane of the pinhole and $h$ is the distance of the point source from the plane of the pinhole [15].

6) Reconstruction algorithm: A direct compensating GPU reconstruction algorithm was developed for multi-pinhole micro-SPECT imaging specifically. This ray-tracing algorithm consists of a pixel-driven forward projector and a voxel-driven back projector. These are combined in the OSEM algorithm. Resolution recovery was based on a multi-ray approach using 7 weighted rays [12]. These 7 rays intersect the circular opening of the pinhole in a hexagonal pattern with one of the points at the centre, and are weighted accordingly. This effectively subsamples the pinhole diameter.

All SPECT datasets were reconstructed to $60 \times 60 \times 60$ voxels with $1 \times 1 \times 1 \mathrm{~mm}^{3}$ voxel size. All reconstructions were stopped at 10 iterations with 8 subsets. This leads to a maximum reconstruction time of 2 minutes per dataset.

\section{Image analysis}

The calibration phantom allows us to convert reconstructed image counts to $\mathrm{MBq} / \mathrm{ml}$ through a scaling factor SF:

$$
\mathrm{SF}\left(\frac{M B q}{\text { counts } \times m l}\right)=\frac{A}{T C \times V},
$$

with $A$ the activity of the calibration syringe measured in $\mathrm{MBq}$ in the dose calibrator (Veenstra VDC-404, Veenstra Instruments, Joure, Netherlands), $T C$ the total number of counts in the reconstructed image, and $V$ the volume of one voxel in milliliters.

$T C$ was measured as the sum of the values in a regionof-interest (ROI) around the hot part of the syringe in the reconstructed SPECT image of the calibration phantom (see Fig. 2a), to minimize the influence of noise contributions and reconstruction artifacts to SF. For ${ }^{111} \mathrm{In}$, both photopeak reconstructions were first summed before determining the SF.

All images were analyzed in the open-source software package AMIDE ${ }^{1}$. The ROIs per vial were chosen sufficiently small to minimize the influence of the PVE. An example is shown on Fig. 2b. The mean activity obtained from these ROIs was then plotted against the activity obtained from the dose

\footnotetext{
${ }^{1}$ Amide's a Medical Imaging Data Examiner, http://amide.sourceforge.net/
} 
TABLE III: Summary of quantification errors after all corrections.

\begin{tabular}{|l|c|c|c|}
\hline & $1: 4$ & $1: 2$ & $1: 1$ \\
\hline${ }^{99 m} \mathrm{Tc}$ & $-24.41 \%$ & $-5.14 \%$ & $-1.46 \%$ \\
${ }^{125} \mathrm{I}$ & $-15.17 \%$ & $-10.85 \%$ & $-20.96 \%$ \\
${ }^{111} \mathrm{In}$ & $-9.93 \%$ & $-2.34 \%$ & $-5.59 \%$ \\
\hline
\end{tabular}

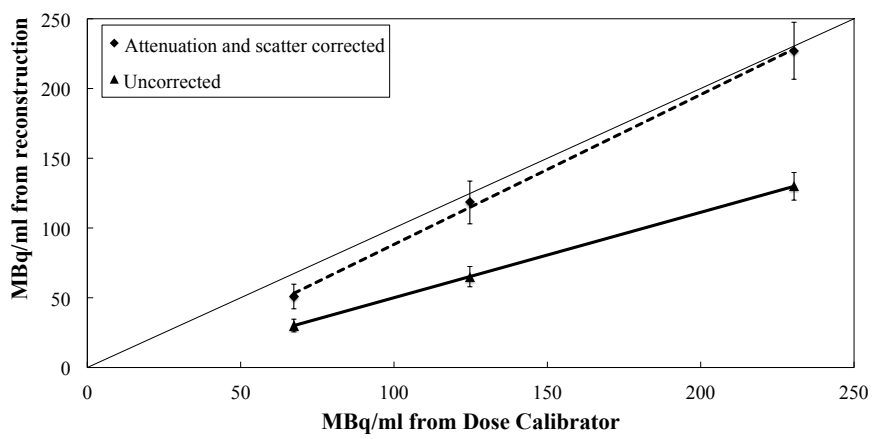

Fig. 3: Relationship between measured activity concentration and reconstructed activity concentration for ${ }^{99 m} \mathrm{Tc}$ (mean $\pm \mathrm{SD})$.

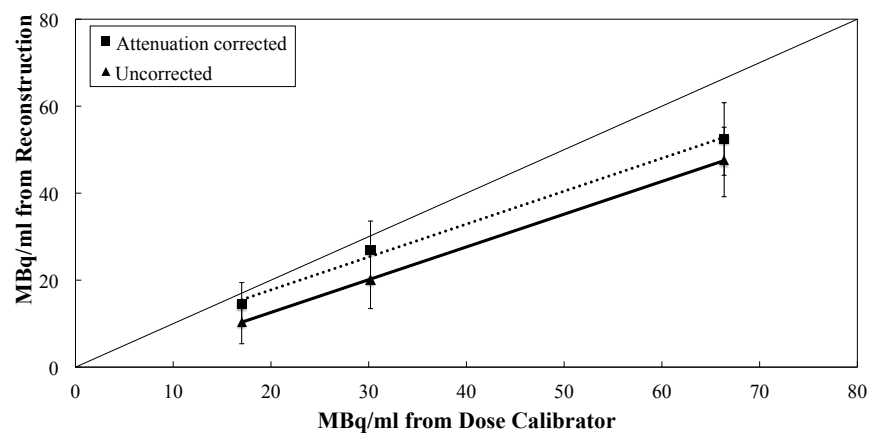

Fig. 4: Relationship between measured activity concentration and reconstructed activity concentration for ${ }^{125} \mathrm{I}($ mean $\pm \mathrm{SD})$.

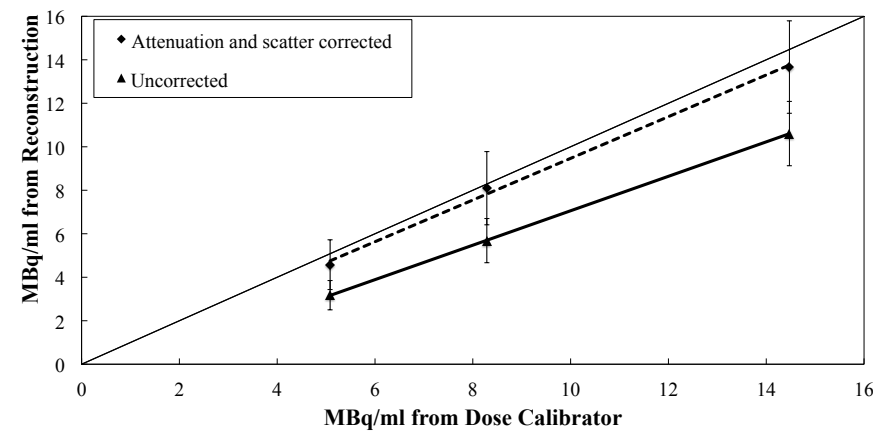

Fig. 5: Relationship between measured activity concentration and reconstructed activity concentration for ${ }^{111} \mathrm{In}($ mean $\pm \mathrm{SD}$ ).

calibrator, with the standard deviation from each ROI as error bars per data point.

\section{RESULTS}

Fig. $2 \mathrm{a}$ and $2 \mathrm{~b}$ show the reconstructed images of the calibration syringe and the 3 -vial phantom, obtained with ${ }^{99 m} \mathrm{Tc}$. Fig.
3 shows the relationship between the measured concentration and the reconstructed activity concentration for ${ }^{99 m} \mathrm{Tc}$. The quantification error is largest for larger activity concentrations when no attenuation and scatter correction is applied. This can be alleviated completely for the highest concentration, but shows only a slight improvement for the 1:4 diluted vial.

For ${ }^{125}$ I (Fig. 4), only attenuation correction was applied. A small increase in quantification accuracy can be noted for all three datapoints, with little increase in standard deviation on the mean absolute activity. The quantification error is lower for lower activity levels.

Fig. 5 shows the relation for ${ }^{111} \mathrm{In}$. The quantification error is significantly reduced by applying all correction methods.

Table III summarizes the relative quantification errors for all three isotopes when all corrections were applied during reconstruction.

\section{DISCUSSION}

Fig. 3, 4 and 5 show that absolute quantification is possible on the FLEX Triumph system. For ${ }^{99 m} \mathrm{Tc}$ a relative error of $1.46 \%$ can be noted for the undiluted vial. Both scatter and attenuation correction have significantly reduced the quantification error. Both diluted vials (1:2 and 1:4) show a significantly higher quantification error, with the error for the 1:4 diluted vial exceeding the standard deviation. The slope of the corrected trend line is higher than the slope of the ground truth line. This may be due to wrong scatter correction due to misestimation at very high count rates, as scatter correction changes the slope of the trend line. This will be further investigated in the future.

For ${ }^{125} \mathrm{I}$, no scatter correction was implemented. Hwang et al. [17] have shown that the scatter-to-primary ratio (SPR) can rise up to $30 \%$ in ${ }^{125}$ I. The quantification error is the highest in the undiluted vial, as SPR is linearly dependent on activity. We were unable to implement scatter correction (see Section II-C1) for ${ }^{125}$ I because of the bad energy resolution at low energies, making it impossible to measure the scatter fraction in the photopeak.

Furthermore, a small mismatch between the attenuation map generated from the $\mu \mathrm{CT}$ scan and the attenuation as seen by ${ }^{125} \mathrm{I}$ will theoretically lead to an underestimation of the attenuation in the 1:2 and 1:4 vials. The iodine CT contrast agent will exhibit a K-edge at $33 \mathrm{keV}$, very close to the $35 \mathrm{keV}$ ${ }^{125}$ I peak. The CT reconstruction will always underestimate the attenuation as the X-ray spectrum is polyenergetic, and will not be able to show the K-edge attenuation precisely. This effect can however not be measured when scatter is not corrected for. The same effect will only be of minor importance for ${ }^{99 m} \mathrm{Tc}$ due to its relatively higher photopeak energy than what is used for the CT scan. As our setup does not allow for good validation of this effect, we will include proper validation in upcoming work.

Lastly, for ${ }^{111} \mathrm{In}$, only a small quantification error is noted. The small error is due to quantification errors inside the high photopeak window (seperate plots are not shown). This may be due to penetration of the high energy photons through the Tungsten collimator, as there were much lower errors inside 
the low photopeak window. The K-edge of the iodine-based contrast agent has little effect here, due to the high energy photopeaks in ${ }^{111} \mathrm{In}$.

\section{Conclusion}

We have developed a GPU-based micro-SPECT reconstruction algorithm using state-of-the-art techniques available from literature, able to reconstruct absolute quantitative microSPECT images on a preclinical multi-pinhole system. The absolute quantification is best when both attenuation and scatter correction are applied, together with resolution recovery.

In the future, we will further improve on the attenuation correction and scatter correction, and use Monte Carlo simulations to model the scatter of ${ }^{125} \mathrm{I}$, as this scatter could not be accurately measured using CZT detectors. Furthermore, we will investigate if the K-edge of iodine can be regarded as an extra degrading effect.

\section{REFERENCES}

[1] P. Almeida et al., Absolute quantitation of iodine-123 epidepride kinetics using single-photon emission tomography: comparison with carbon-11 epidepride and positron emission tomography, Eur J Nucl Med Mol Imaging, vol. 26, no. 12, pp. 1580-1588, 1999.

[2] C. M. Finucane, I. Murray, J. K. Sosabowski, J. M. Foster and S. J. Mather, Quantitative Accuracy of Low-Count SPECT Imaging in Phantom and In Vivo Mouse Studies, Int J Mol Imaging, vol. 2011, pp. $1-8,2011$.

[3] C. Vanhove, M. Defrise, A. Bossuyt and T. Lahoutte, Improved quantification in single-pinhole and multiple-pinhole SPECT using micro-CT information, Eur J Nucl Med Mol Imaging, vol. 36, no. 7, pp. 10491063, 2009.

[4] C. Wu et al., Absolute quantitative total-body small-animal SPECT with focusing pinholes, Eur J Nucl Med Mol Imaging, vol. 37, no. 11, pp. 2127-2135, 2010.

[5] M. Holstensson, C. Hindorf, M. Ljungberg, M. Partridge and G. D. Flux, Optimization of Energy-Window Settings for Scatter Correction in Quantitative 111In Imaging: Comparison of Measurements and Monte Carlo Simulations, Cancer Biotherapy and Radiopharmaceuticals, vol. 22, no. 1, pp. 136-142, 2007.

[6] P. K. Leichner et al., Patient-specific dosimetry of indium-111- and yttrium-90-labeled monoclonal antibody CC49, J Nucl Med, vol. 38, no. 4, pp. 512-516, 1997.

[7] T. Funk et al., A new CdZnTe-based gamma camera for high resolution pinhole SPECT, IEEE NSS Conference Record, 2004.

[8] D. Bequé, J. Nuyts, G. Bormans, P. Suetens and P. Dupont, Characterization of acquisition geometry of pinhole SPECT. IEEE Trans Med Imag, vol. 22, no. 5, pp. 599-612, 2003.

[9] D. Bequé, J. Nuyts, P. Suetens and G. Bormans, Optimization of geometrical calibration in pinhole SPECT, IEEE Trans Med Imag, vol. 24, no. 2, pp. 180-190, 2005.

[10] K. Ogawa, Y. Harata, T. Ichihara, A. Kubo and S. Hashimoto, A practical method for position-dependent Compton-scatter correction in single photon emission CT, IEEE Trans Med Imag, vol. 10, no. 3, pp. 408-412, 1991.

[11] E. Kint, S. Staelens and S. Vandenberghe, Five energy window scatter correction for ${ }^{111}$ In microSPECT, SNM Abstracts, vol. 50, no. 2, pp. 1469, 2009.

[12] C. Vanhove, A. Andreyev, M. Defrise, J. Nuyts and A. Bossuyt, Resolution recovery in pinhole SPECT based on multi-ray projections: a phantom study, Eur J Nucl Med Mol Imaging, vol. 34, no. 2, pp. 170$180,2007$.

[13] R. Accorsi and S. D. Metzler, Analytic determination of the resolutionequivalent effective diameter of a pinhole collimator, IEEE Trans Med Imag, vol. 23, no. 6, pp. 750-763, 2004.

[14] H. O. Anger, Radioisotope cameras, Instrumentation in Nuclear Medicine, G. J. Hine, Ed. New York: Academic, vol. 1, pp. 485-552, 1967.

[15] J. R. Mallard and M. J. Myers, The performance of a gamma camera for the visualization of radioactive isotopes in vivo, Phys Med Biol, vol. 8, pp. $165-182,1963$.
[16] M. A. King, D. J. Devries, T. S. Pan, P. H. Pretorius and J. A. Case, An investigation of the filtering of TEW scatter estimates used to compensate for scatter with ordered subset reconstructions, IEEE Trans Nucl Sci, vol. 44, pp. 1140-1145, 1997.

[17] A. B. Hwang, B. L. Franc, G. T. Gullberg and B. H. Hasegawa, Assessment of the source of error affecting the quantitative accuracy of SPECT imaging in small animals, Phys Med Biol, vol. 53, pp. 2233-2252, 2008. 